\section{PARENTERAL SYMPTOMS AND COMPLICATIONS IN CHILDREN WITH \\ INFLAMMATORY BOWEL DISEASES IN \\ RELATION TO THE MUTATIONS OF CARD15 GENE}

U.E. Grzybowska-Chlebowczyk ${ }^{1}$, H. Wos ${ }^{1}$, A. Sieron ${ }^{2}$, M. Kajor ${ }^{3}$, S. Wiecek ${ }^{1}$, H. KoryciakKomarska², A. Auguściak-Duma²

${ }^{1}$ Department of Paediatrics, ${ }^{2}$ Department of General, Molecular Biology and Genetics, ${ }^{3}$ Department of Pathomorphology, Silesian Medical University, Katowice, Poland

The aim of the study was the evaluation of parenteral symptoms and complications incidence in children with non-specific colitis and their analysis in relation to the mutations of CARD15 gene.

Patients and methods: The study involved 38 children with Crohn's disease, aged from 5 to18 years and 40 children with ulcerative colitis, aged from 6 to18 years.

The control group included 23 children, aged from 4 to 18 years, with functional disorders of the alimentary tract resulting from lactose intolerance.

In all the examined patients mutations R702W, G908R and L1007fs of CARD15 gene were determined, according to the protocol described by Tukel.

Results: Parenteral symptoms in the group of children with Crohn's disease, manifested as arthritis and erythema nodosum, were observed in 7 patients $(18.4 \%)$, whereas in the group with ulcerative colitis- in 4 children $(10 \%)$. Intestinal complications in the form of stenosis, fistula, abscess and gastrointestinal bleeding were the most frequently observed in children with Crohn's disease $15(39.5 \%)$

Parenteral symptoms were statistically significantly more frequent in children with Crohn's disease and at least one mutation of CARD15 gene.

Intestinal complications statistically appeared more often in children with Crohn's disease and mutation L1007fs.

\section{Conclusions:}

1. Parenteral symptoms and intestinal complications more frequently occur in the group of children with Crohn's disease, in comparison with the children with ulcerative colitis.

2. We observed a relation between parenteral symptoms and at least one mutation of CARD15 gene, and a relation between intestinal complications and L1007fs mutation.

\section{4}

\section{ACUTE ACALCULOUS CHOLECYSTITIS IN CHILDREN: A 10-YEAR RETROSPECTIVE STUDY IN A SINGLE CENTER}

$$
\text { S.-C. Huang }{ }^{1} \text {, Y.-J. Yang }{ }^{2}
$$

${ }^{1}$ Department of Pediatrics, Kuo General Hospital, ${ }^{2}$ Department of Pediatrics, National Cheng Kung University and Hospital, Tainan, Taiwan R.O.C.

Objective: The aims of this study were to define the etiology, clinical presentation, and prognosis of acute acalculous cholecystitis (AAC) in children.

Methods: Children aged less than 18 years diagnosed with AAC were analyzed retrospectively between 2000 and 2009. The demographic and clinical characteristics, etiology, and outcome were recorded. AAC was defined as GB wall thickness of greater than $3.5 \mathrm{~mm}$ and duration of symptoms less than 1 month. The severity of sonographic findings were scored, with 1 point each given for wall thickness greater than $3.5 \mathrm{~mm}$, GB distention, sludge, and pericholecystic fluid.

Results: A total of 109 children (male/female $=1.18$, mean age $=5.8$ years) were diagnosed with AAC. The most common presentation was fever $(88 \%)$, followed by hepatomegaly (73\%) and jaundice (49\%). $65 \%$ and $72 \%$ of patients had thrombocytopenia and elevated alanine aminotransferase, respectively. The common causative etiologies were infectious diseases (58\%) and systemic illnesses (27\%). In addition to the wall thickening, the most frequent sonographic finding was GB distension (34\%), followed by sludge (15\%), and pericholecystic fluid $(14 \%)$. All of our patients were treated nonoperatively. Sixteen (15\%) patients died. Children with mortality had a significantly higher rate of shock $(p<0.001)$, anemia $(p=0.01)$, thrombocytopenia $(p=0.04)$, hypofibrinogenemia $(p=0.002)$, presence of pericholecystic fluid, and higher sonographic scores $(p=0.04)$ than those with survival. 